\title{
THEORETICAL LIGHT CURVES OF RECURRENT NOVAE
}

\author{
M. KATO \\ Department of Astronomy, Keio University, Yokohama 223, Japan
}

\begin{abstract}
Theoretical light curves for the decay phase of recurrent novae are obtained using optically thick wind theory. With the OPAL opacity tables the decay time scale is drastically shortened.
\end{abstract}

Theoretical light curves for the decay phase of recurrent novae are presented. We have used OPAL new opacity tables (Rogers and Iglesias 1992: private communication) which has a large peak about 2 or 3 times larger than the old opacity around $T=2 \times 10^{5} \mathrm{~K}$ due to iron lines. This enhancement of the opacity causes strong mass loss (optically thick winds) and then the evolutional time-scale is drastically shortened. The decay time scale of recurrent novae strongly depends on the white dwarf mass and weakly depends on the hydrogen content of the envelope with solar composition of heavy element. From the fitting of theoretical light curve with observed data, I have found that the white dwarf mass is about $1.35 M_{\odot}$ for $\mathrm{U}$ Sco, $1.0 M_{\odot}$ for $\mathrm{T}$ Pyx, and $1.15 M_{\odot}$ for RS Oph. As well as optical data, UV light curve is useful to determine the white dwarf mass from fitting of theoretical light curves with observational data.

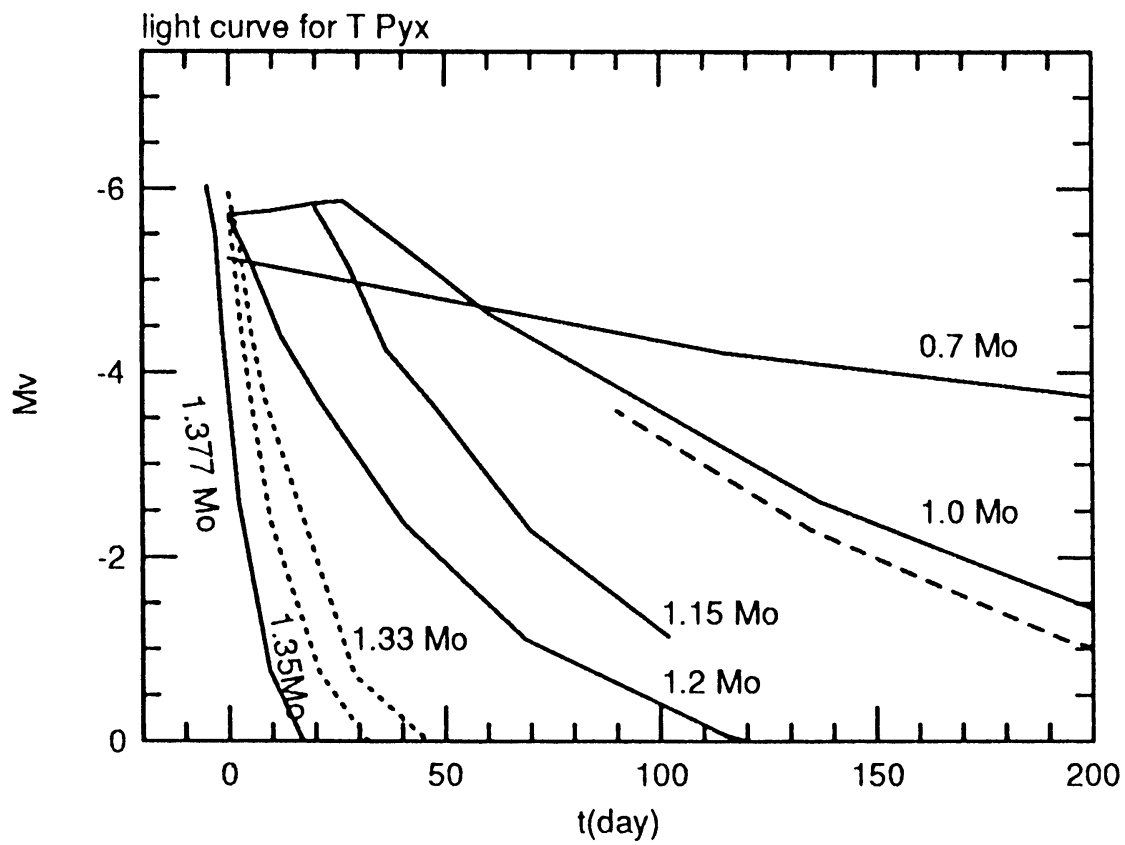

Fig. 1. Theoretical light curves for the decay phase of recurrent novae. White dwarf masses are given near each curve. Thick curve: $X=0.7$, dotted: $X=0.5$, and dashed: $X=0.35$ while $Z=0.02$ are assumed for all models. 